\title{
Displacement and Stress Distribution of the Craniomaxillofacial Complex Under Different Surgical Conditions: A Three-Dimensional Finite Element Analysis of Fracture Mechanics
}

\author{
Junjie Chen \\ Central South University \\ Yuhan Xu \\ Central South University \\ Chengri Li \\ Central South University \\ Lingling Zhang \\ Central South University
}

Fang Yi

Central South University

Yanqin Lu ( $\sim 213031 @ c s u . e d u . c n$ )

Central South University

\section{Research Article}

Keywords: Maxillary transverse deficiency, Osteotomy-assisted arch expansion, Finite element analysis, palatal suture, cone-beam computed tomography.

Posted Date: September 28th, 2021

DOI: https://doi.org/10.21203/rs.3.rs-936447/v1

License: (c) (i) This work is licensed under a Creative Commons Attribution 4.0 International License. Read Full License

Version of Record: A version of this preprint was published at BMC Oral Health on November 22nd, 2021. See the published version at https://doi.org/10.1186/s12903-021-01941-1. 


\section{Original Research}

Displacement and stress distribution of the craniomaxillofacial complex under different surgical conditions: a three-dimensional finite element analysis of fracture mechanics

Junjie Chen ${ }^{1, *}$, Yuhan $\mathrm{Xu}^{1}{ }^{1}$, Chengri Li ${ }^{1}$, Lingling Zhang ${ }^{1}$, Fang Yi, Yanqin $\mathrm{Lu}^{1, \#}$

1. Hunan Key Laboratory of Oral Health Research \& Human 3D Printing Engineering Research Center of Oral Care \& Human Clinical Research Center of Oral Major Diseases and Oral Health \& Xiangya Stomatological Hospital \& Xiangya School of Stomatology, Central South University, Changsha, Hunan 410008, China

\section{*Junjie Chen is the first author of this research}

\#Corresponding author: Yanqin Lu,

Hunan Key Laboratory of Oral Health Research \& Human 3D Printing Engineering Research Center of Oral Care \& Human Clinical Research Center of Oral Major Diseases and Oral Health \& Xiangya Stomatological Hospital \& Xiangya School of Stomatology, Central South University, Changsha, Hunan 410008, China

E-mail:213031@csu.edu.cn 


\section{Abstract}

Objective: To provide a simplified treatment strategy for patients with maxillary transverse deficiency. We investigated and compared the fracture mechanics and stress distribution of a midline palatal suture under dynamic loads during surgically-assisted rapid palatal expansion.

Methods: Based on the cone-beam computed tomography (CBCT) data of a 21-year-old female volunteer, a three-dimensional model of the craniomaxillofacial complex (including the palatal suture) was constructed. A finite element analysis model was constructed based on meshwork. After the yield strength of the palatal suture was set, an increasing expansion force $(0-500 \mathrm{~N})$ was applied within $140 \mathrm{~ms}$ to calculate the time-load curve, which mimicked nonsurgical bone expansion (model A). The same method was used to evaluate the fracture process, time and stress distribution of the palatal suture in maxillary lateral osteotomy-assisted (model B) and LeFort osteomy I (LFIO)-assisted expansion of the maxillary arch (model C).

Results: Compared with model A, the palatal suture of model B and model C showed a faster stress accumulation rate and shorter fracture time, and the fracture time of model $B$ and model $C$ was almost identical. Compared with model $A$, we discovered that model $B$ and model $C$ showed greater lateral extension of the maxilla, and the difference was reflected mainly in the lower part of the maxilla, and there was no difference between model B and model $\mathrm{C}$ in lateral extension of the maxilla.

Conclusions: Compared with arch expansion using nonsurgical assistance (model A), arch expansion using maxillary lateral wall-osteotomy (model B) or LFIO had a faster rate of stress accumulation, shorter time of fracture of the palatal suture and increased lateral displacement of the maxilla. Compared with arch expansion using LFIO (model C), arch expansion using lateral osteotomy (model B) had a similar duration of palatal suture rupture and lateral maxillary extension. In view of the trauma and serious complications 
associated with LFIO, maxillary lateral wall-osteotomy could be considered a substitute for LFIO.

\section{Keywords}

Maxillary transverse deficiency, Osteotomy-assisted arch expansion, Finite element analysis, palatal suture, cone-beam computed tomography. 


\section{Background}

Maxillary transverse deficiency is a common deformity in adolescents and adults. It can cause transverse maxillomandibular discrepancies and posterior crossbite. ${ }^{1-3}$ Surgically assisted rapid palatal expansion (SARPE) has become the primary choice for palatal expansion in adults, ${ }^{4-7}$ but there are controversies regarding the choice of surgical method. ${ }^{8-10} \mathrm{~A}$ LeFort I osteotomy (LFIO) can relieve maxillofacial resistance and concentrate the expansion force, and is used commonly for SARPE to accelerate palatal expansion. $5,7,11$

A LFIO in SARPE has some disadvantages: the side-effects of general anesthesia, risk of fracture, prolonged recovery period and an injury risk to the pterygopalatine segment of the maxillary artery. ${ }^{12-14}$

A simplified surgical method has been considered to replace the LFIO. Glassman and colleagues undertook conservative surgery on 16 adults for palatal expansion, and obtained an excellent therapeutic effect. ${ }^{15}$ Antilla and coworkers reported the feasibility and long-term stability of lateral osteotomyassisted maxillary expansion. ${ }^{16}$ Recent research has demonstrated the necessity of a paramedian osteotomy and pterygomaxillary separation in partial- and complete-fusion sutures. ${ }^{17}$ However, the conclusions of those clinical or basic-research studies were limited by small sample sizes, absence of a control group and lack of biomechanical research.

The finite element analysis (FEA) method was first used to evaluate the mechanical behavior of skeletal parts in 1972. The FEA method is noninvasive, convenient and repeatable. We postulated that a threedimensional (3D) FEA method could be used to simulate a surgical procedure 
by weakening the influence and stress of craniofacial-bone resistance and fracture of the palatal raphe. In this way, biomechanical effects could be investigated.

We wished to establish a 3D FEA model of the craniomaxillofacial complex based on fracture mechanics. Then, we aimed to use this modeling method to simulate nonsurgical-assisted, maxillary lateral osteotomy-assisted and LFIOassisted expansion of the maxillary arch (hereafter termed "arch"). We compared the time required for fracture of the palatal suture, the distribution of craniomaxillofacial stress and the change in displacement of the maxillary complex when arch expansion had been completed. In this way, a theoretical basis for simplified surgery could be provided.

\section{Materials and methods}

\subsection{Reconstruction of a 3D FEA mesh model of the craniomaxillofacial} complex

This study was conducted using the cone-beam computed tomography (CBCT) data $\left(0.300-\mathrm{mm}\right.$ layer; voxel size, $\left.0.463 \times 0.463 \times 0.300 \mathrm{~mm}^{3}\right)$ of a 21-year-old female volunteer diagnosed with maxillary transverse deficiency and deciduous tooth retention. This study was approved by the Ethics Committee of Xiangya School of Stomatology. CBCT sections were saved as digital imaging and Communications in Medicine images, and then imported to E-3D v16.22 (Hunan, China) for 3D reconstruction. Then, the STL model was exported to Geomagic studio v12 (3D Systems, Rock Hill, SC, USA) in which noise is eliminated from the geometry and the contours are smoothed. The gray value ranged from $200 \mathrm{HU}$ to $2000 \mathrm{HU}$, and hard tissue was selected for reconstruction. In the reconstruction software, the bone cortex and cancellous bone were not distinguished. However, the bone cortex was denoted as $1 \mathrm{~mm}$ from the base of cancellous bone. Poisson's ratio and the elastic modulus were set in the FEA to distinguish the bone cortex and 
cancellous bone. Then, 3D models with different osteotomies were imported into Hypermesh ${ }^{\mathrm{TM}}$ (Altair, Frisco, TX, USA), which was used to create the FEA models. The resulting FEA models (Figure 1a) comprised an average of 245,516 elements and 45,585 nodes. The palatal cleft contained 1207 nodes and 3302 solid tetrahedral units. This modeling method was used to simulate three methods of palatal expansion: nonsurgical-assisted (model A); lateral osteotomy-assisted (model B); LFIO-assisted (model C). A schematic diagram of surgical incisions is shown in Figure $1 \mathrm{~b}-\mathrm{d}$.

\subsection{Material parameters, boundary conditions and load setting}

The displacement and rotation of the nodes around the foramen magnum in $\mathrm{X}$, $\mathrm{Y}$ and $\mathrm{Z}$ directions were set as 0 . The treatment method of palatal expansion was simulated. Four nodes of area $1.8 \mathrm{~mm} \times 5 \mathrm{~mm}$ were selected from the palate to be loaded with a horizontal force that was increased from $0 \mathrm{~N}$ to 500 $\mathrm{N}$ within $140 \mathrm{~ms}$ (Figure 1e).

The material parameters of the tooth, cortical bone, cancellous bone and midline palatine suture were set according to former research (Table 1). The material at the midline palatine suture was set as MAT-Plastic-Kinematic, and the yield strength was $1 \mathrm{MPa}$. That is, when the stress applied to the material reached the yield strength that we set, its mesh disappeared gradually to simulate crack propagation until the material broke.

Table 1. Properties of the materials used in our simulation

$$
\text { Young's modulus (MPa) Poisson's ratio }
$$

\begin{tabular}{lll}
\hline Cortical bone & 13700 & 0.30 \\
Cancellous bone & 1370 & 0.30 \\
Tooth & 19890 & 0.31
\end{tabular}


The time required for the palate to reach yield strength (T1), crack initiation (T2) and final fracture (T3) were recorded. Three marking points were selected to evaluate the lateral displacement of the maxillary body after palatal expansion. The marking points are shown in Figure $1 \mathrm{f}$.
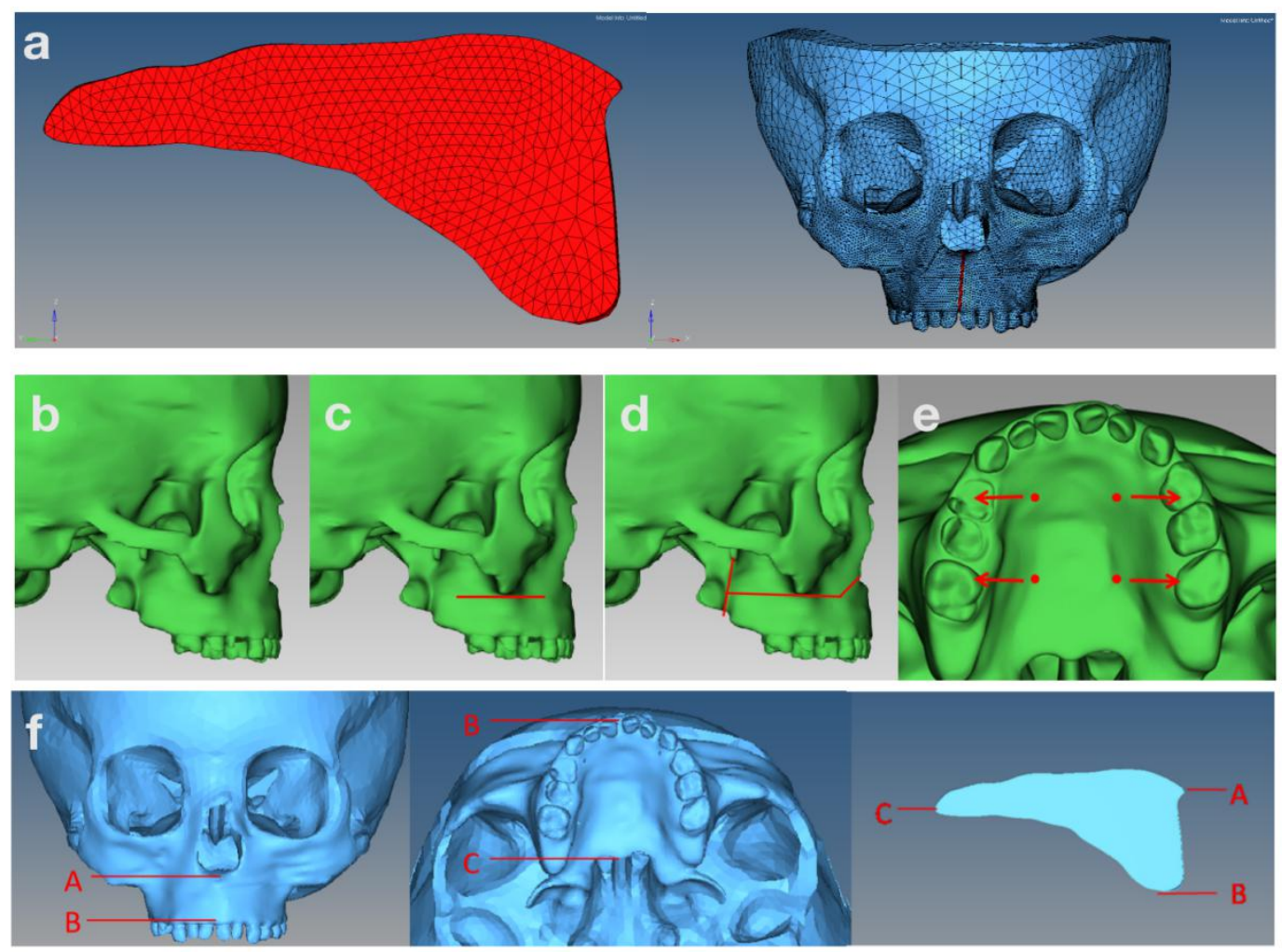

Figure 1. Reconstruction and process of a three-dimensional finite elements analysis of a mesh model. (a) Craniomaxillofacial complex and meshwork of the palatal suture. (b) Nonsurgical-assisted arch expansion. (c) Lateral osteotomy-assisted maxillary palatal expansion. (d) LeFort I osteotomyassisted maxillary palatal expansion. (e) Loading positions. (f) Red arrows denote measurement positions.

\subsection{Volunteer patient}


The volunteer was a 21-year-old woman with chief complaints of malocclusion, crossbite and deciduous tooth retention. Intraoral examination revealed deciduous tooth retention, and mixed dentition with class-III malocclusion. The overjet was $-5 \mathrm{~mm}$ and overbite was $-3 \mathrm{~mm}$. The dental formula is shown in Figure 2.

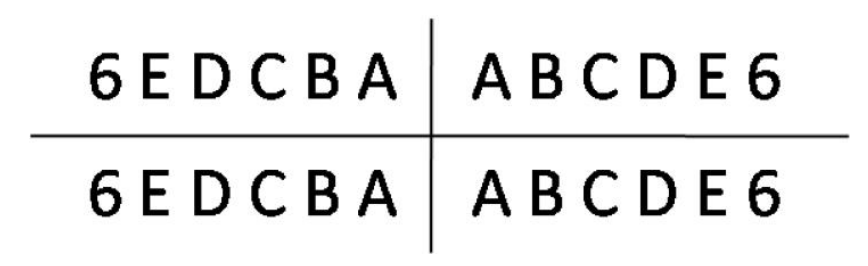

Figure 2. The dental formula of our volunteer.

Miniscrew-assisted rapid palatal expansion (MARPE) involves application of a force directly to the maxilla using miniscrews and a skeletal anchorage expander. MARPE was selected for skeletal maxillary expansion. However, the volunteer's palatal suture was fused. In addition, patients with cleidocranial dysplasia have been reported to have more dense and compact alveolar bone, which indicates that their facial skeleton provides greater resistance to expansion than that of healthy people. Accordingly, MARPE failed to overcome greater resistance or an open, fused midline palatal suture after 30 days of treatment.

According to FEA results, lateral osteotomy-assisted maxillary palatal expansion was selected, and was completed under local anesthesia in a clinic. Hence, corticotomy-facilitated MARPE was deemed to be the most suitable treatment modality. With regard to the maxilla expander, a custom-made bone-borne device was newly designed to cut costs and reduce invasion. Hence, a new method of corticotomy-facilitated MARPE was developed to resolve maxillary dysplasia while minimizing the side-effects of the procedure. Our treatment plan combined surgery and modified techniques to meet the requirements of our volunteer. The procedure was designed to be more 
$\mathrm{B}$

efficacious and less invasive. The patient accepted the option of corticotomy-facilitated MARPE (Figure 3).
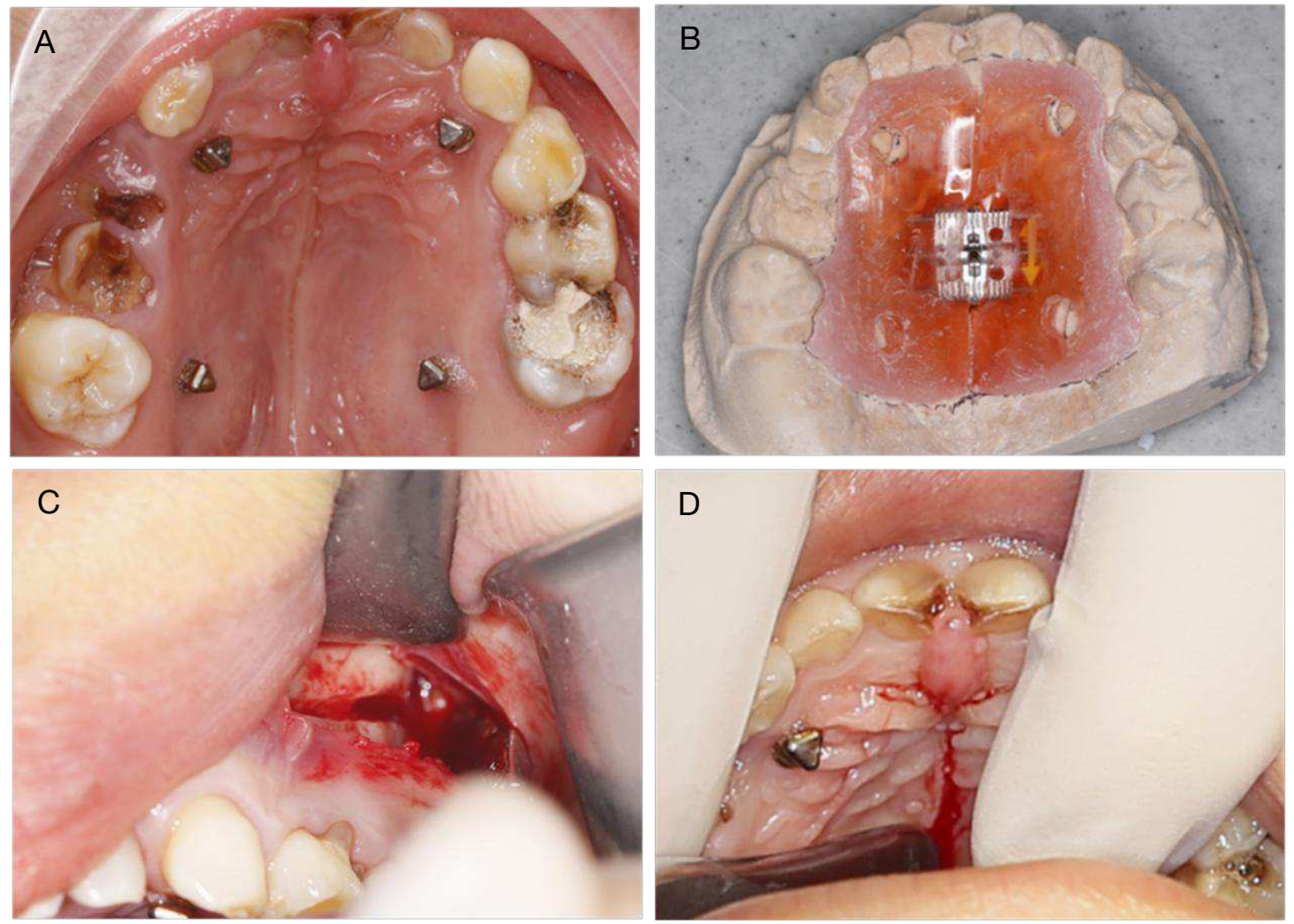

Figure 3. Miniscrew-assisted rapid palatal expansion (MARPE). (A)

Fixation of four miniscrews in the palate. (B) Custom-made appliance. (C) Lateral cortiotomy. (D) Mid-palatal cortiotomy.

\section{Results}

\subsection{Fracture process of the midline palatal suture}

The fracture process of the midline palatal suture was similar among the three models. The nonsurgical-assisted palatal expansion model was selected to analyze the process based on fracture mechanics.

From $0 \mathrm{~ms}$ to $52 \mathrm{~ms}$ (stress-accumulation stage), the stress on the palate increased gradually until the yield strength was reached (Figure 4). From 52 to $68 \mathrm{~ms}$, plastic deformation occurred in the midline palatal suture, and the 
yield stress no longer increased. At $69 \mathrm{~ms}$, an initial crack occurred in the posterior inferior portion because some elements began to erode. From $69 \mathrm{~ms}$ to $102 \mathrm{~ms}$, the initial cracks in the front and lower parts began to expand backward and upward, which represents the process of crack propagation. At $102 \mathrm{~ms}$, the suture was totally fractured. From $102 \mathrm{~ms}$ to $140 \mathrm{~ms}$, displacement of the craniomaxillofacial complex was increased until the end of the expansion.

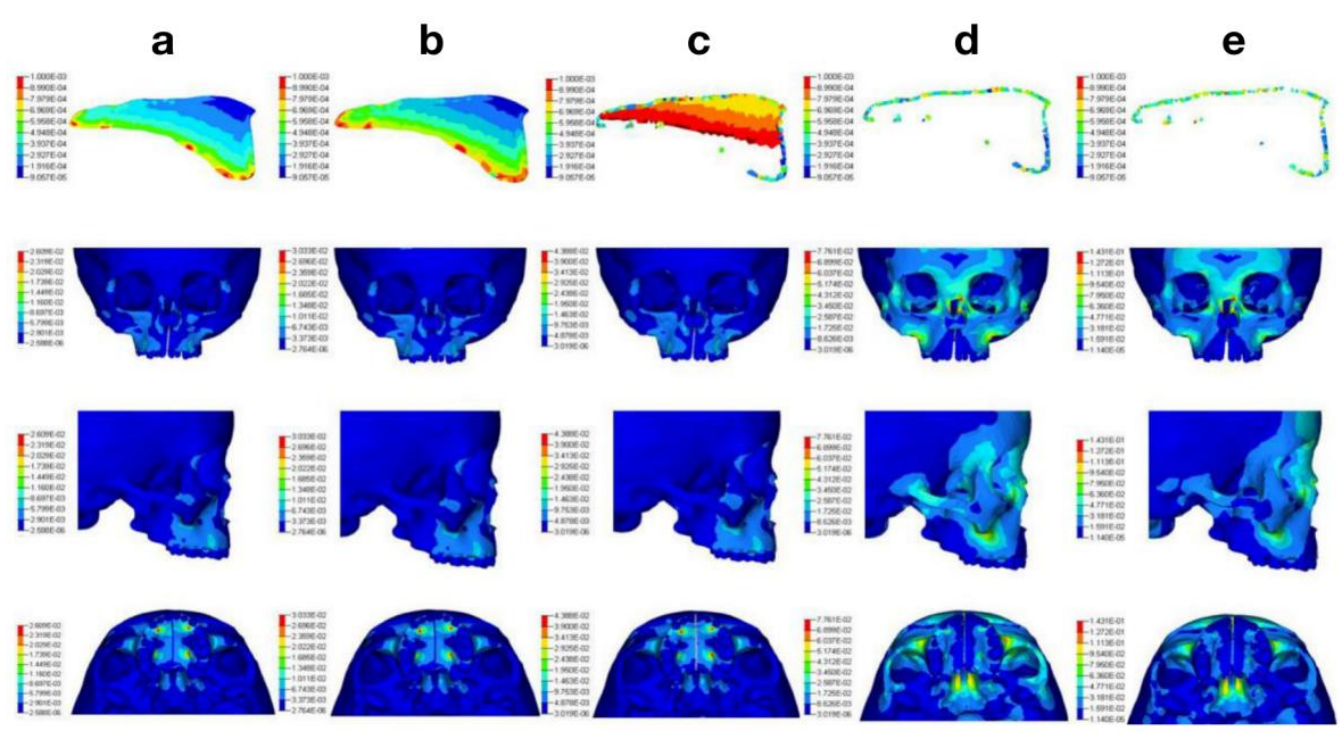

Figure 4. Fracture of the palatal suture and stress distribution of the craniomaxillofacial complex in nonsurgical-assisted arch expansion. (a) The lower mesh before palatal suture reached the yield stress at $52 \mathrm{~ms}$. (b) At 68 ms, the lower mesh began to disappear. (c) The crack continued to propagate upwards and backwards at $80 \mathrm{~ms}$. (d) At $102 \mathrm{~ms}$, the midline palatal suture was completely fractured and the material lost all continuity. (e) At $140 \mathrm{~ms}$, a load was no longer present.

Stress accumulation and the fracture velocity between different models were compared. The rate at which stress accumulated in the palatal suture was faster in model $B$ and model $C$ than that in model A. At $30 \mathrm{~ms}$, stress in the palatal suture was greater in model $B$ and model $C$ than that in model $A$ 
(Figure 5). At $60 \mathrm{~ms}$, crack initiation in model A was absent, whereas crack propagation began in model $B$ and model $C$. At $90 \mathrm{~ms}$, a crack was observe in model $A$, whereas the palatal suture was totally fractured in model $B$ and model C. None-surgical
(model A)

lateral osteotomy (model B)

Lefort 1 surgery (model C)
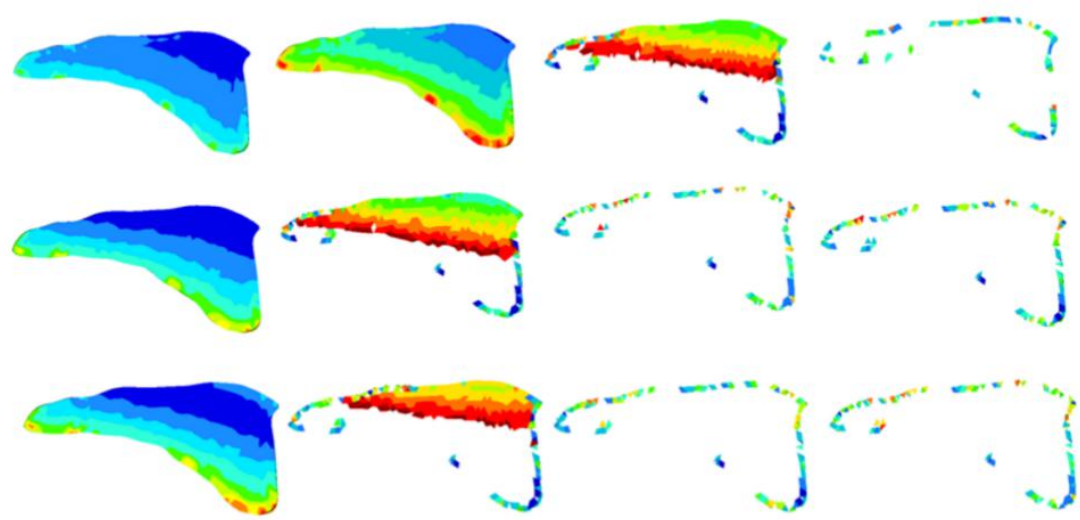

$30 \mathrm{~ms}$

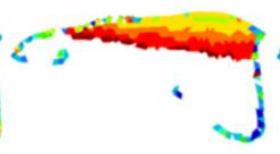

$60 \mathrm{~ms}$

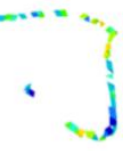

$90 \mathrm{~ms}$

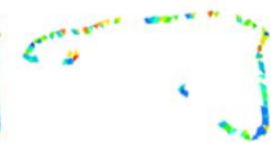

$120 \mathrm{~ms}$

Figure 5. Comparison of the fracture process of the palatal suture between models $A, B$ and $C$.

The time point of the yield strength (T1), crack initiation (T2) and complete fracture (T3) was compared among the three models. As shown in Table 2, the non-surgical group required the longest time for palatal suture fracture. The lateral osteotomy-assisted and LFIO-assisted group had the similar palatal suture fracture rate.

T1

Model A

Model B
52

36
T2

68

47
70

T3

102 
Table 2. Timings (T1, T2, T3) in models $A, B$ and $C(\mathrm{~ms})$

\subsection{Craniomaxillofacial stress and strain distribution}

Before fracture of the palatal suture, for the nonsurgical group, a significant concentration of stress on the zygomatic alveolar ridge was noted. For the lateral osteotomy group and LFIO group, there was a significant stress reduction on the zygomatic crest due to the surgical incision, whereas a concentration of stress occurred on the surgical-incision edge (Figure 6).
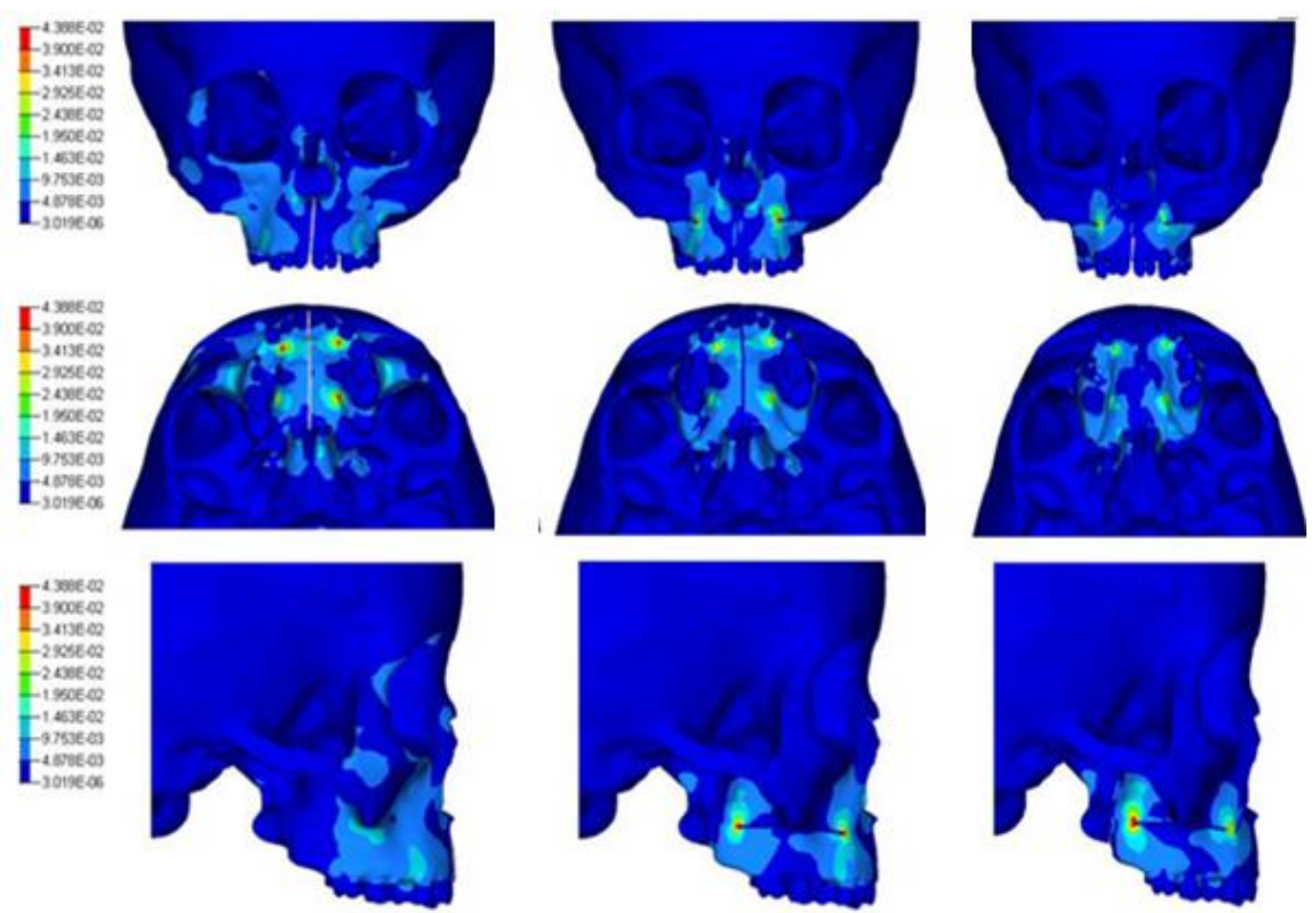

Figure 6. Distribution of craniomaxillofacial stress in models A, B and C before fracture of the middline palatal suture. 


\subsection{Lateral displacement of the maxilla}

Comparison of lateral displacement of the maxilla is shown in Table 3. From the coronal direction, the maxilla of the three groups presented trapezoidal expansion. The LFIO group had the largest expansion in the anterior and posterior parts.

Table 3. Lateral displacement of the measure points $(A, B, C)$ with models $A, B$ and $\mathrm{C}(\mathrm{mm})$

\begin{tabular}{llll}
\hline & A & B & C \\
\hline Model A & 2.17 & 2.93 & 1.92 \\
Model B & 2.26 & 3.93 & 2.10 \\
Model C & 2.40 & 4.24 & 2.24 \\
\hline
\end{tabular}

\subsection{Treatment result for our volunteer}

A good result was obtained after two expansions. Specifically, after the first expansion, extra space was not observed between the two maxillary deciduous central incisors. Then, CBCT was undertaken to ascertain if the treatment plan was efficacious and realizable. CBCT after first maxillary expansion revealed a crack at the middle-posterior part of the midline palatal suture in transverse section, and the crack extension had reached the nasal septum in the coronal plane (Figure 7). 

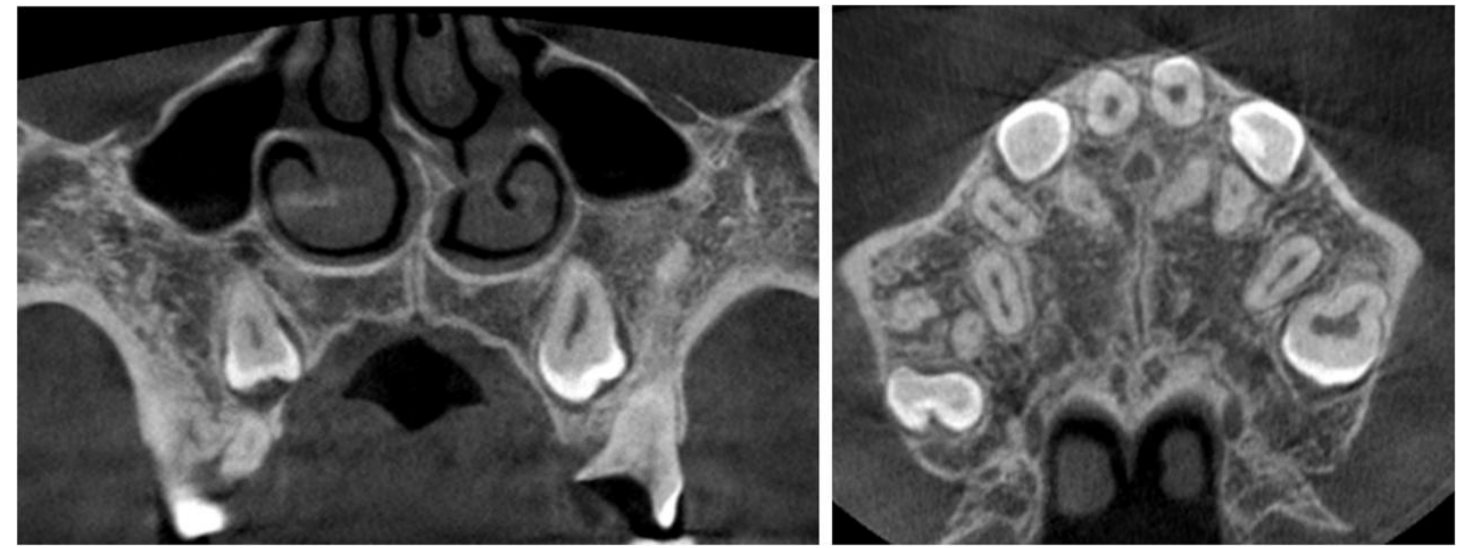

Figure 7. CBCT after MARPE after the first maxillary expansion

We suspected that stress accumulation was not sufficient to completely expand the midline palatal suture, and that an additional maxilla expander was needed. After the second expansion, the space of the primary central incisor was $4 \mathrm{~mm}$, and posterior crossbite had improved. After 1 month, CBCT was carried out again: the midline palatal suture was completely cracked from front to back and from bottom to top. As a result, the fracture was approximately parallel in the transverse plane, and V-shaped in the front plane (Figure 8).
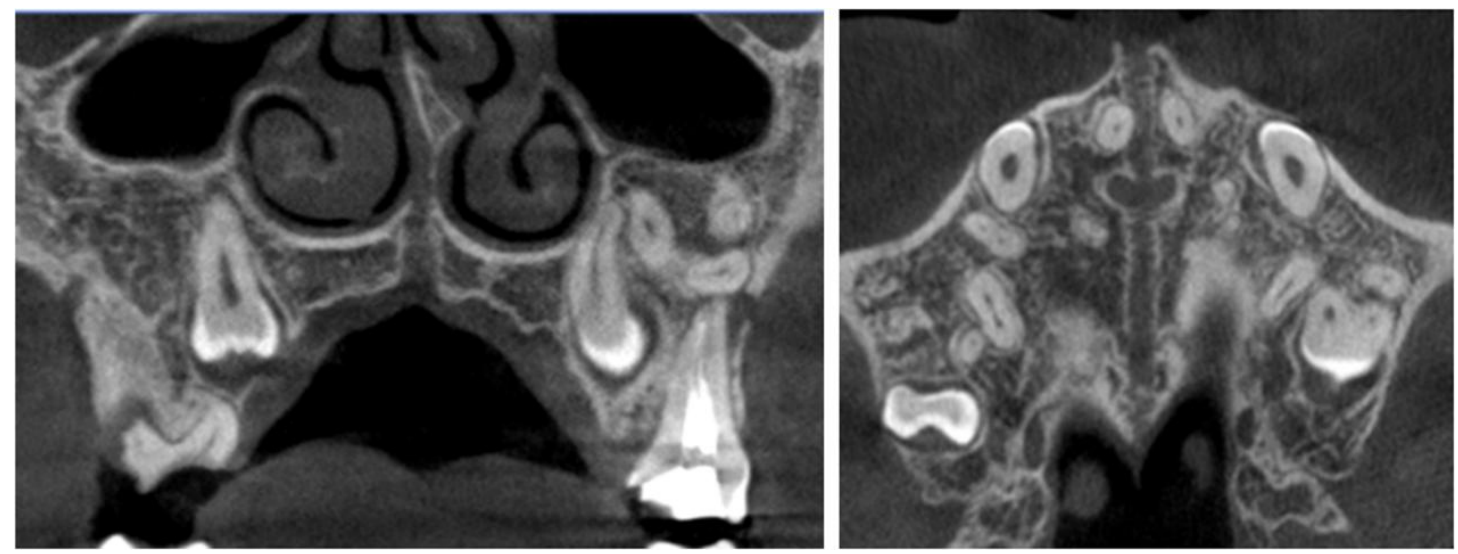

Figure 8. CBCT images 1 month after MARPE.

\section{Discussion}

SARME is used commonly to correct maxillary deficiency in adults, but consensus on the choice of surgical method is lacking. ${ }^{4,5}$ The traditional LFIO 
can fully weaken the resistance to arch expansion. However, it necessitates surgery under general anesthesia, which leads to surgical trauma and can lead to complications. ${ }^{18-20}$ Osteotomy of the lateral maxillary wall can be superior to the LFIO. It can be undertaken under local anesthesia in the outpatient setting to release the lateral maxillary wall only (i.e., the area around the zygomatic alveolar crest) to reduce resistance to arch expansion.

Figure 4 shows the initiation and propagation of cracks in the region of the palatal fissure after model $A$ had been subjected to a continuous expansion force in the arch. Eventually, macroscopic cracks formed to cause fractures in the palatal suture area, thereby achieving the therapeutic effect of bony expansion. This type of fracture follows the process of crack initiation, crack propagation and material fracture under a continuous load. Figure 6 reflects the fracture process of the palatal fissure in three models. Under an identical loading condition, the fracture process of the palatal suture in model B and model $\mathrm{C}$ developed faster than that in model $\mathrm{C}$. These results suggested that the palatal suture in the surgical groups accumulated a greater expansion force due to a reduction in resistance of craniomaxillofacial bone. This hypothesis explains why it is difficult to expand the palatal suture in adult patients using traditional RME, but it can be achieved using SARME. ${ }^{17,} 21$ Table 2 shows a direct comparison of the fracture rate of the palatal suture. The time required for the surgical groups to fracture the palatal suture was significantly shorter than that for the nonsurgical group. Hence, a surgical procedure can realize a reduction of expansion resistance and accelerated fracture of the palatal suture. ${ }^{9,10,22}$ However, the results for model B were almost identical to those of model $\mathrm{C}$, indicating that even a simple incision in the lateral wall of the maxilla could weaken the resistance of the craniomaxillofacial bone and accelerate fracture of the midline palatal suture.

With regard to the stress distribution of the three models (Fig. 4), in the 
nonsurgical group, the stress concentration was in the anterior, lateral and posterior walls of the maxilla. However, the stress in the zygomatic alveolar ridge was relatively high, indicating that the lateral wall of the maxilla may be the most important source of arch-expansion resistance, and that accelerated fracture of the palatal suture in the lateral wall-osteotomy group could confirm this conjecture.

The sphenoid body in the three models had a high concentration of stress. This region has extremely important structures, such as the trigeminal nerve and middle meningeal artery. Lanigan and colleagues reported on skull base (SB) fractures and ruptures of the middle meningeal artery caused by surgically assisted arch expansion. ${ }^{23}$ Those complications could be related to the complex structure of the SB and excessive stress accumulation during arch expansion. Using 3D FEA, Holberg and coworkers simulated a large wing of the sphenoid bone moving outwards by $2 \mathrm{~mm}$, and found that the stress on the SB increased significantly. ${ }^{24}$

We showed that the stress distribution in the SB remained high even after incision of the pterygomaxillary junction. This phenomenon may have occurred because the maxilla continued to have a connection with the surrounding bones even in SARME using LFIO. For example, the inner wall of the maxilla and outer wall of the nasal cavity have extensive connections, so the stress of arch expansion can be transmitted to the SB. To fully protect important SB structures, a segmental maxillary osteotomy can be considered, whereby the maxilla is disconnected completely from the surrounding bone, allowing the maxilla to move freely.

Studies have considered the posterior wall of the maxilla to be one of the main resistance areas during arch expansion. 5,12,25 To achieve greater arch expansion, the posterior connection of the maxilla must be truncated. 
However, some clinical studies in recent years have proffered different opinions. In 2014, Sygouros and colleagues collected 20 cases of SARME for retrospective analyses: 10 cases were truncated and the remaining 10 cases were not. ${ }^{26}$ They found no significant difference in maxillary dilatation between the two groups, but the group with a untruncated maxillary junction showed more buccal inclination in the posterior tooth segment. Zandi and collaborators conducted a double-blind clinical randomized controlled trial in 2016. Thirty patients were divided into two groups and treated with SARME, and the variable was whether the maxillary connection was detached from the broken wing. ${ }^{26}$ Changes in the osseous and dental properties of the maxilla in the short-term after SARME were evaluated and compared. There was no significant difference in the mean expansion volume between the two groups.

We hope to not only accelerate fracture of the midline palatal suture through appropriate surgery, but also to increase expansion of the upper jaw. Table 3 shows that lateral displacement in the lower maxilla of the lateral wallosteotomy group was larger than that of the nonsurgical group, and slightly smaller than that of the LFIO group. Hence, "ideal" maxillary expansion could be achieved even if the lateral wall-osteotomy of the maxilla was straightforward. Anttila and coworkers selected 20 patients (mean age $=31$ years) with lateral osteotomy-assisted RME of the arch. ${ }^{27}$ After arch expansion, the mean width between canines and molars increased by 4.2-7.1 $\mathrm{mm}$. Two years later, the mean width between canines and molars decreased by $0.5-1.3 \mathrm{~mm}$, thereby achieving a stable and ideal therapeutic effect.

Our study had two main limitations. First, compared with the tetrahedral element, the hexahedral element can improve the accuracy of calculation to a certain extent. However, the craniofacial anatomic structure is complex, and the tetrahedral mesh has better adaptability to complex geometry. In the future, we can consider replacing the tetrahedral element with the hexahedral 
element to improve the calculation accuracy. Second, in the application of isotropy and anisotropy in finite elements, although the results simulated by anisotropy are more realistic, medical biomechanics uses isotropy primarily, and relevant reference points are needed. In the future, we can combine micro-CT and isotropy for investigations.

\section{Conclusions}

Our study elicited three main findings. First, compared with arch expansion using nonsurgical assistance (model A), arch expansion using maxillary lateral wall-osteotomy (model B) or LFIO had a faster rate of stress accumulation, shorter time of fracture of the palatal suture and increased lateral displacement of the maxilla. Second, compared with arch expansion using LFIO (model C), arch expansion using lateral osteotomy (model B) had a similar duration of palatal suture rupture and lateral maxillary extension. Third, in view of the trauma and serious complications associated with LFIO, maxillary lateral wall-osteotomy could be considered a substitute for LFIO.

\section{Ethics approval and consent to participate}

The study design was approved by the Ethical Committee of Xiangya School of Stomatology. All participants gave informed consent and consent was written. No individual person's data were included in this research.

\section{Availability of data and materials}

The datasets used and/or analysed during the current study is available from the corresponding author on reasonable request.

\section{Funding}

This work was supported by the Science and Technology Department of Hunan Province, China [grant number XCSZ2019, No.74]. 


\section{Authors' contributions}

Junjie Chen wrote this manuscript, performed the clinical treatment and built

the 3D model.Yuhan Xu and Chengri Li performed the finite elements analysis. Lingling Zhang and Fang Yi collected the relative data. Yanqin Lu designed the whole project and supervised the therapeutic process.

\section{Acknowledgments}

Not applicable

\section{Consent for publication}

The study design was approved by the Ethical Committee of Xiangya School of Stomatology. All participants gave informed consent and consent was written. No individual person's data were included in this research.

\section{Conflict of interest}

Authors declare there are no competing interests, financial and non-financial, in relation to the work described.

\section{References}


1. Pedro, P. V., Eric, R. T. \& Francisco, V. D. M. Effects of Surgically Assisted Rapid Maxillary Expansion On the Modification of the Pharynx and Hard Palate and On Obstructive Sleep Apnea, and their Correlations. Journal of Cranio-Maxillo-Facial Surgery. 48, (2020).

2. Panpan, L. et al. Changes in Maxillary Width and Upper Airway Spaces in Young Adults After Surgically Assisted Rapid Palatal Expansion with Surgically Facilitated Orthodontic Therapy. Oral Surgery, Oral Medicine, Oral Pathology and Oral Radiology. 127, (2019).

3. M, H. S. C. et al. Effect of the Pterygomaxillary Disjunction On Surgically Assisted Rapid Palatal Expansion in Context of Orthodontic Treatment. Oral surgery, oral medicine, oral pathology and oral radiology. 130, (2020).

4. Audrey, Y., Christian, G., Soroush, Z. \& Stanley, Y. L. Distraction Osteogenesis Maxillary Expansion (DOME) for Adult Obstructive Sleep Apnea Patients with Narrow Maxilla and Nasal Floor. Sleep Med. 65, (2020).

5. Nikhil, G., A., S. J. \& Pratik, K. S. The Stability of Surgically Assisted Rapid Maxillary Expansion (SARME): A Systematic Review. Journal of Cranio-Maxillo-Facial Surgery. 48, (2020).

6. Prado, G. P. R., Pereira, M. D., Biló, J. P. R., Furtado, F. \& Ferreira, L. M. Stability of Surgically Assisted Rapid Palatal Expansion: A Randomized Trial. J. Dent. Res. 92, (2013).

7. Paolo, S., Raul, V., Georges, H. \& Bertrand, J. Maxillary Expansion Using Transpalatal Distraction in Patients with Unilateral Cleft Lip and Palate. Plast. Reconstr. Surg. 119, (2007).

8. M, H. S. C. et al. Immediate Dental and Skeletal Influence of Distractor Position On Surgically Assisted Rapid Palatal Expansion with Or without Pterygomaxillary Disjunction. Int. J. Oral Max. Surg. (2020).

9. Akbari, M., Prabhu, R., Khanna, S. \& Turner, M. D. Resident Commentary: Is there a Significant Difference in Relapse and Complication Rate of Surgically Assisted Rapid Palatal Expansion Using Tooth-Borne, BoneBorne, and Orthodontic Mini-Implant-Borne Appliances (Ploder Et Al, 2020)? J Oral Maxillofac Surg. 79, e1-e3 (2021).

10. Ploder, O. et al. Is there a Significant Difference in Relapse and Complication Rate of Surgically Assisted Rapid Palatal Expansion Using Tooth-Borne, Bone-Borne, and Orthodontic Mini-Implant-Borne Appliances? Journal of oral and maxillofacial surgery : official journal of the American Association of Oral and Maxillofacial Surgeons. 79, (2021).

11. H., B., M., E., L., E. \& S., R. M. New Technique for Midpalatal Osteotomy in Surgically-Assisted Rapid Palatal Expansion. British Journal of Oral and Maxillofacial Surgery. 55, (2017).

12. Lee, S. C. et al. Effect of Bone-Borne Rapid Maxillary Expanders with and without Surgical Assistance On the Craniofacial Structures Using Finite Element Analysis. American journal of orthodontics and dentofacial orthopedics : official publication of the American Association of 
Orthodontists, its constituent societies, and the American Board of Orthodontics. 145, (2014).

13. Rina, H., Yukiho, K., Michiko, T., Tatsuo, K. \& Keiji, M. Long-Term Orthodontic and Surgical Treatment and Stability of a Patient with Beckwith-Wiedemann Syndrome. American Journal of Orthodontics \& Dentofacial Orthopedics. 145, (2014).

14. Kohei, N. et al. Orthodontic-Surgical Approach for Treating Skeletal Class III Malocclusion with Severe Maxillary Deficiency in Isolated Cleft Palate. The Cleft Palate-Craniofacial Journal. 56, (2019).

15. Glassman, A. S., Nahigian, S. J., Medway, J. M. \& Aronowitz, H. I. Conservative Surgical Orthodontic Adult Rapid Palatal Expansion: Sixteen Cases. Am J Orthod. 86, 207-213 (1984).

16. Anttila, A. et al. Feasibility and Long-Term Stability of Surgically Assisted Rapid Maxillary Expansion with Lateral Osteotomy. Eur J Orthod. 26, 391395 (2004).

17. Shi, Y., Zhu, C. N. \& Xie, Z. Displacement and Stress Distribution of the Maxilla Under Different Surgical Conditions in Three Typical Models with Bone-Borne Distraction: A Three-Dimensional Finite Element Analysis. J. Orofac. Orthop. 81, 385-395 (2020).

18. Seblain, D. et al. Minimally Invasive Versus Standard Approach in LeFort 1 Osteotomy in Patients with History of Cleft Lip and Palate. J Stomatol Oral Maxillofac Surg. 119, 187-191 (2018).

19. van de Lande, L. S. et al. Surgical Correction of the Midface in Craniofacial Microsomia. Part 1: A Systematic Review. J Craniomaxillofac Surg. 46, 1427-1435 (2018).

20. Pluijmers, B. I. et al. Part 2: Is the Maxillary Canting and its Surgical Correction in Patients with CFM Correlated to the Mandibular Deformity? J Craniomaxillofac Surg. 46, 1436-1440 (2018).

21. Dias, R. R., Takeshita, W. M., Sverzut, A. T., Trivellato, A. E. \& Sverzut, C. E. Linear Analysis of the Nasal Septum in Patients Treated with Surgically Assisted Rapid Maxillary Expansion. Am J Orthod Dentofacial Orthop. 159, 71-80 (2021).

22. Rachmiel, A., Turgeman, S., Shilo, D., Emodi, O. \& Aizenbud, D. Surgically Assisted Rapid Palatal Expansion to Correct Maxillary Transverse Deficiency. Ann Maxillofac Surg. 10, 136-141 (2020).

23. Lanigan, D. T. \& Mintz, S. M. Complications of Surgically Assisted Rapid Palatal Expansion: Review of the Literature and Report of a Case. J Oral Maxillofac Surg. 60, 104-110 (2002).

24. Holberg, C., Steinhauser, S. \& Rudzki-Janson, I. Rapid Maxillary Expansion in Adults: Cranial Stress Reduction Depending On the Extent of Surgery. Eur J Orthod. 29, 31-36 (2007).

25. Kufta, K., Melean, L. P., Grady, M. S. \& Panchal, N. Massive Middle Cerebral Artery Infarction After Surgically Assisted Rapid Palatal Expansion: A Case Report. J Oral Maxillofac Surg. 75, 1521-1529 (2017). 
26. Sygouros, A., Motro, M., Ugurlu, F. \& Acar, A. Surgically Assisted Rapid Maxillary Expansion: Cone-Beam Computed Tomography Evaluation of Different Surgical Techniques and their Effects On the Maxillary Dentoskeletal Complex. Am J Orthod Dentofacial Orthop. 146, 748-757 (2014).

27. Anttila, A. et al. Feasibility and Long-Term Stability of Surgically Assisted Rapid Maxillary Expansion with Lateral Osteotomy. Eur J Orthod. 26, 391395 (2004). 